\title{
Re-imaginons les travaux pratiques pour la formation des ingénieurs en électronique
}

\author{
Miguel Ángel García Pérez, Yves Leduc, Fabien Ferrero \\ Pôle CNFM PACA - Polytech’Nice Sophia - Département d'électronique \\ 930, route des Colles - 06903 Sophia Antipolis \\ Contact : miguelangel.gp@me.com - yves.leduc@ polytech.unice.fr - fabien.ferrero@unice.fr
}

\begin{abstract}
Résume :
Notre objectif est d'essayer de combler l'écart actuel entre les travaux traditionnels de laboratoire universitaire et les méthodes réelles de design de circuits intégrés dans l'industrie. En offrant à nos étudiants une première vue globale du système, nous les accompagnons ensuite vers une approche plus pragmatique sur les objectifs en termes de «Puissance, Performance, Surface » de chacune des parties constituantes. L'enseignement est basé sur la compréhension du comportement et des paramètres clés des différentes parties, à travers un cheminement où la modélisation, la simulation, le prototypage et la caractérisation occupent une place fondamentale [1].
\end{abstract}

Mots-clés : circuits mixtes, électronique analogique, PLL, VCO, PFD, modélisation, simulation.

\section{Introduction}

Dans notre philosophie les étudiants devront accomplir la réalisation d'un circuit au plus proche du transistor. Plusieurs niveaux de difficulté leurs sont proposés d'une façon incrémentale : le design d'un oscillateur contrôlé en tension basé sur des inverseurs (VCO) ; un comparateur de phase avec des portes CMOS existantes (PFD) ; une pompe de charges avec son filtre basé sur des transistors bipolaires $(\mathrm{CP}$ et $\mathrm{LF})$; puis l'intégration du système complet, une PLL à pompe de charges, et sa comparaison avec le circuit du commerce existant TI CMOS PLL CD4046B.

Dans cet esprit, il commencera son design par un VCO constitué de composants simples, ce qui va lui permettre de fixer la fenêtre de fréquences d'utilisation. Les autres modules seront également conçus, puis une fois l'assemblage réalisé, l'étude des caractéristiques, aussi bien expérimentale qu'issue de la simulation, donnera lieu à une phase d'optimisation afin d'obtenir les meilleurs performances possibles.

Dans les perspectives proches nous pensons donner un caractère ludique au produit final en intégrant la PLL dans une chaîne de démodulation d'un signal radio FM.

\section{Motivations et objectifs}

Plusieurs raisons nous ont emmené à concevoir nos travaux pratiques avec une philosophie différente. Le désalignement entre les apprentissages standardisés et les besoins industriels en est la principale, particulièrement dans le domaine des circuits intégrés à signaux mixtes, où nos compagnies locales nous ont encouragés à proposer de nouvelles techniques et méthodologies de travail pour mieux préparer nos étudiants. Ceci dit, le processus idéal de conception, fabrication, test et validation d'un circuit intégré complet est trop long et difficilement intégrable de façon efficace dans nos enseignements.

Notre projet est constitué de mini-projets où les étudiants travaillent en autonomie, la nouveauté consiste à proposer un sous-ensemble d'exercices cohérents, tout en s'attaquant à des challenges réels qui couvrent les différents domaines d'intérêt du design de circuits mixtes, et en intégrant systématiquement la modélisation et la simulation dans notre processus de travail. 


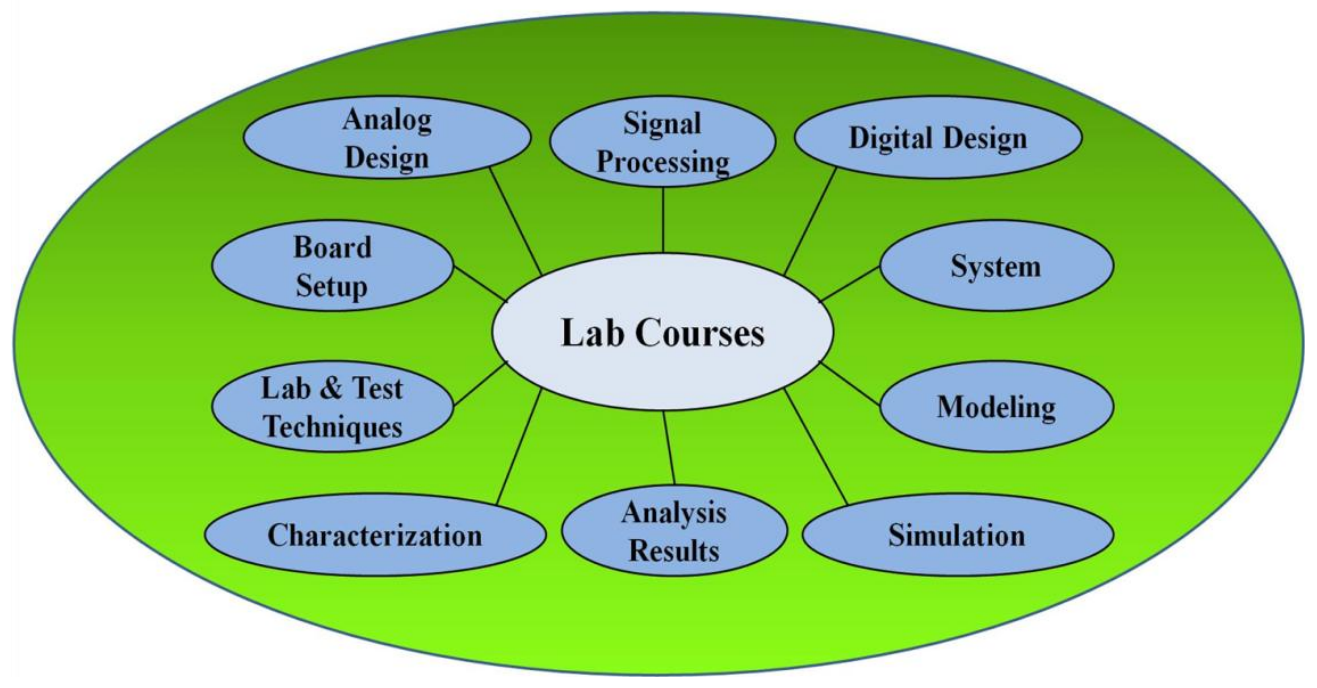

Figure 1 : Ensemble de compétences

Pour ce faire nous avons choisi comme élément d'étude une boucle à verrouillage de phase (PLL) à pompe de charge (CP) [2]. Ce système permettra aux étudiants d'aborder et/ou développer toutes les compétences qu'ils devront maîtriser en fin de cycle (Figure 1).

\section{Structure et fonctionnement d'une CP-PLL}

Une boucle à verrouillage de phase à pompe de charge (CP-PLL) est constituée (Figure 2) d'un comparateur de phase (PFD), qu'est un dispositif séquentiel numérique qui régule le courant fourni par une pompe de charge (CP), laquelle alimente un filtre de boucle (LF), qui converti ce courant en tension analogique, afin de contrôler la fréquence du signal sortant de l'oscillateur contrôlé en tension (VCO). Ce signal est alors rebouclé sur une des entrées du PFD après l'avoir numérisé avec le « clock slicer » $(\mathrm{CSO})$ et divisé par N, pour être comparé avec le signal d'entrée numérisé (CSI) et divisé par M. Ce jeu de divisions nous permettra de générer des signaux analogiques avec un ratio de N/M de la fréquence de référence, ce qui est le fonctionnement de base d'une PLL.

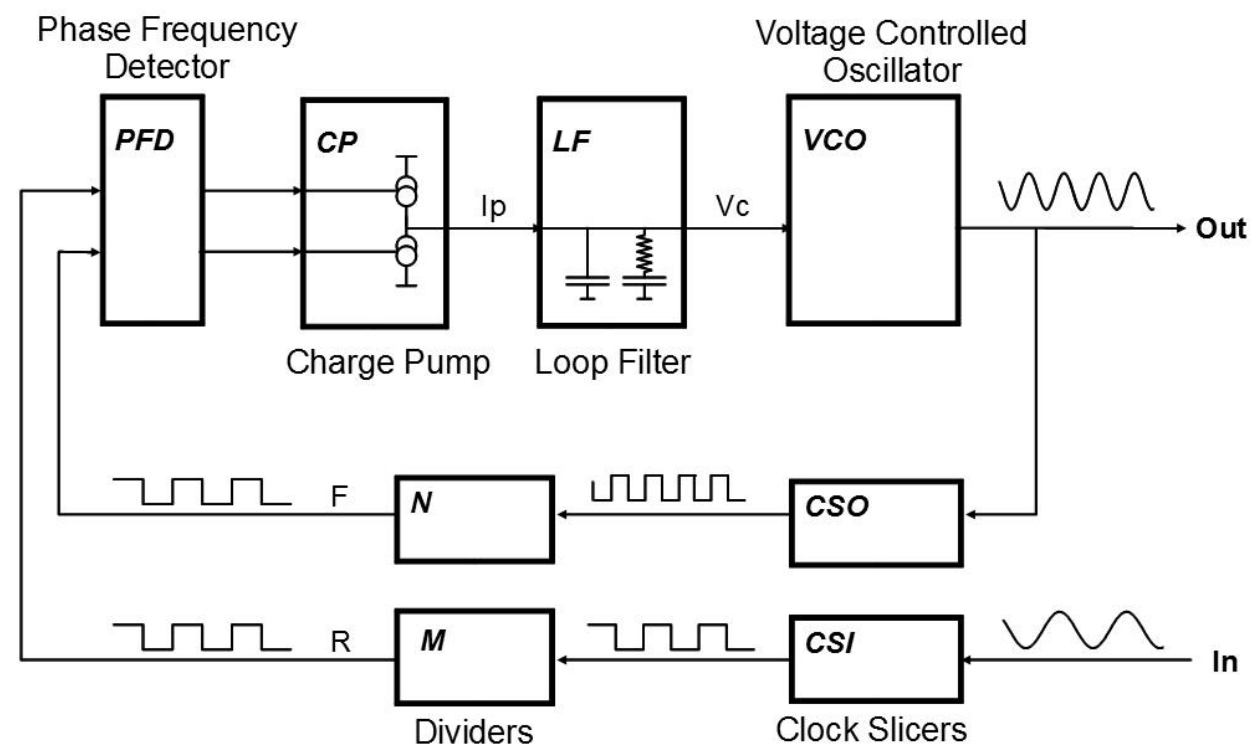

Figure 2 : Schéma bloc CP-PLL 
Dans le cas où le signal de sortie du VCO est trop rapide (ou trop lent), le PFD envoie un signal «moins » (ou «plus ») au CP, qui génère un courant négatif (ou positif) sur le LF, ceci a par conséquence de diminuer (ou augmenter) la tension de contrôle du VCO, ce qui ralenti (ou accélère) le signal de sortie. Si un équilibre est trouvé et le signal se stabilise, la PLL se verrouille.

Plusieurs choix de structure de PFD s'offrent à nos étudiants afin d'atteindre les objectifs recherchés [3]. Le VCO, de son côté, est un des éléments le plus critique d'une CP-PLL, mais peut aussi se construire de manière simple avec un oscillateur en anneau à base d'inverseurs CMOS. Nous leur proposerons diverses alternatives, allant de la combinaison de dispositifs standards discrets jusqu'à des solutions avec de la logique intégrée.

Du fait de sa non-linéarité, il n'est pas surprenant qu'une CP-PLL montre un comportement non trivial, jamais trop loin du chaos [4], ce qui justifiera l'utilisation d'un outil de design tel un simulateur spécifique pour les circuits à signaux mixtes.

\section{Modélisation et simulation}

Le propos de ces travaux pratiques est d'acquérir les compétences appropriées en design pour la micro-électronique, ainsi qu'une connaissance approfondie des circuits à base de PLL. Il est donc important que les étudiants disposent d'un outil de simulation rapide avec des modèles comportementaux précis. Par ailleurs, la séquence de travail complète est synthétisée à travers le diagramme de la figure 3 ci-dessous.

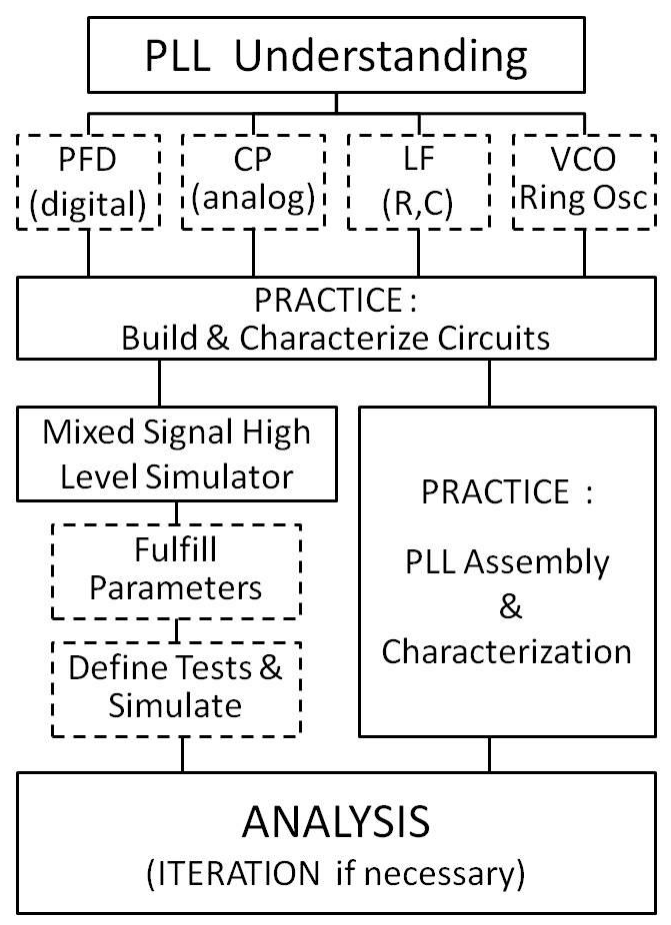

Figure 3 : Séquence globale du TP

Nous avons choisi un modèle haut-niveau commandé par évènement qui a été largement utilisé et prouvé [3] [5]. Ce modèle a été récemment amélioré pour gérer d'avantage de structures génériques. La description la plus appropriée de chaque élément est utilisée :

- le détecteur de phase PFD est une machine d'état numérique. Outre la description fonctionnelle, nous allons modéliser la zone morte du comparateur par 2 délais ajoutés aux évènements venant de la référence ou de la boucle de sortie respectivement. Sa sortie contrôlera la pompe de charge $\mathrm{CP}$, 
- la pompe de charge est un module analogique échantillonné. Il sera modélisé comme un commutateur idéal de sources de courant, contrôlé par le PFD. Sa sortie est modélisée avec une forme d'onde en escalier,

- le filtre de boucle étant un élément passif, il est modélisé comme un circuit linéaire dans le domaine temps. Sa fonction de transfert sera un polynôme dans le domaine de Laplace. La sortie du LF sera obtenue par un outil de transformation numérique inversé de Laplace,

- la fonction de transfert du VCO décrit la fréquence de sortie en fonction de la tension d'alimentation. Il s'agit d'une fonction non-linéaire, mais elle pourra être décrite comme une suite enchainée de fonctions linéaires. Il est possible alors de construire la sinusoïde résultante à partir d'un modèle de modulation de fréquence simple. Du bruit et de la distorsion peuvent y être ajoutés,

- les « clock slicers » CSI et CSO transforment les signaux analogiques en évènements numériques, qui sont les entrées des diviseurs numériques $\mathrm{M}$ et $\mathrm{N}$, lesquels sont modélisés dans le domaine numérique.

Les performances d'une CP-PLL dépendent des paramètres clés suivants :

- PFD : Nombre d'états de la machine d'état

Délai entre le signal de référence et la sortie

Délai entre le signal rebouclé et la sortie

- CP : Nombre et valeurs du courant de sortie

- LF : Fonction de transfert dans le domaine de Laplace

- VCO : Suite de fonctions linéaires de la fréquence en fonction de la tension

- CS : Niveaux de décision

- DIV : rapports de division $\mathrm{M}$ et $\mathrm{N}$

Deux exemples de PFD/CP sont montrés sur la figure 4. Ils diffèrent sur le nombre d'états du PFD (3 ou 5) mais ont le même nombre de niveaux de courant (I-, I0, I+).

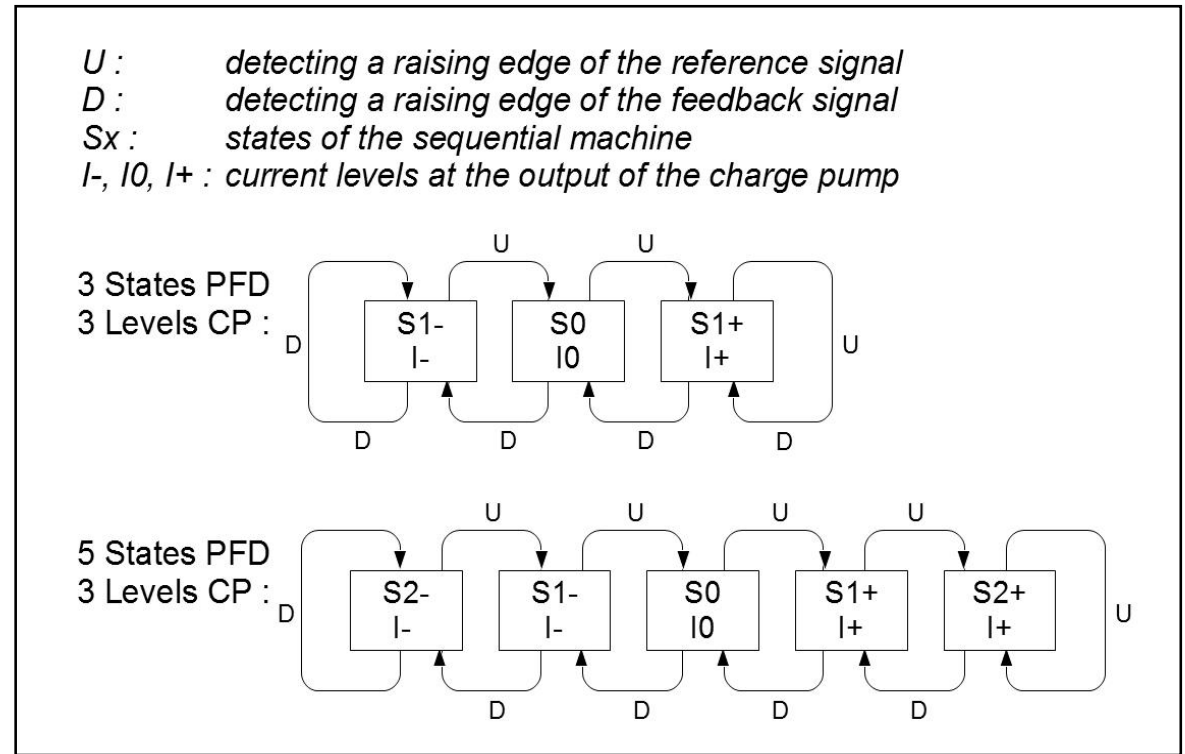

Figure 4 : Combinaison PFD/CP 
Pour intégrer la description de la PLL au sein d'un circuit à signaux mixtes plus important, il nous a été nécessaire de construire un modèle comportemental de haut niveau qui soit compatible avec un simulateur à commande par cycle. Un tel simulateur doit supporter un défilement régulier de données compatibles avec une analyse FFT à une très grande vitesse de simulation. Le simulateur NAPA [6] pour circuits mixtes, écrit et supporté par un des auteurs, répond parfaitement à ces besoins. Il a été récemment mis à disposition de la communauté enseignante par Texas Instruments Inc. et peut être téléchargé gratuitement [7]. NAPA permet de générer des exécutables pour Windows 64 bits, qui sont utilisables directement par les étudiants, de façon à pouvoir tourner des simulations sans pour autant devoir suivre une formation sur le fonctionnement complet de l'outil.

L'adaptation d'un modèle géré par évènements avec un simulateur à commande par cycle n'est pas évidente. Afin de simplifier le problème nous supposons que le signal de référence est déjà numérisé et synchronisé sur l'horloge (cycle) du simulateur (voir figure 5). La sortie du VCO, quand à elle, est asynchrone vis-à-vis du cycle d'horloge du simulateur. Pour une meilleure précision, la simulation tournera à une plus grande vitesse. L'état du PFD sera stable pendant plusieurs cycles (cas 1). Lors d'un évènement synchrone déclenché par la référence en début de cycle, l'état du PFD sera actualisé et se maintiendra sur le cycle suivant (cas 2). Quand l'évènement est commandé par la sortie du VCO, il peut avoir lieu au milieu du cycle et le PFD actualisera alors son état de manière asynchrone (cas 3 ).

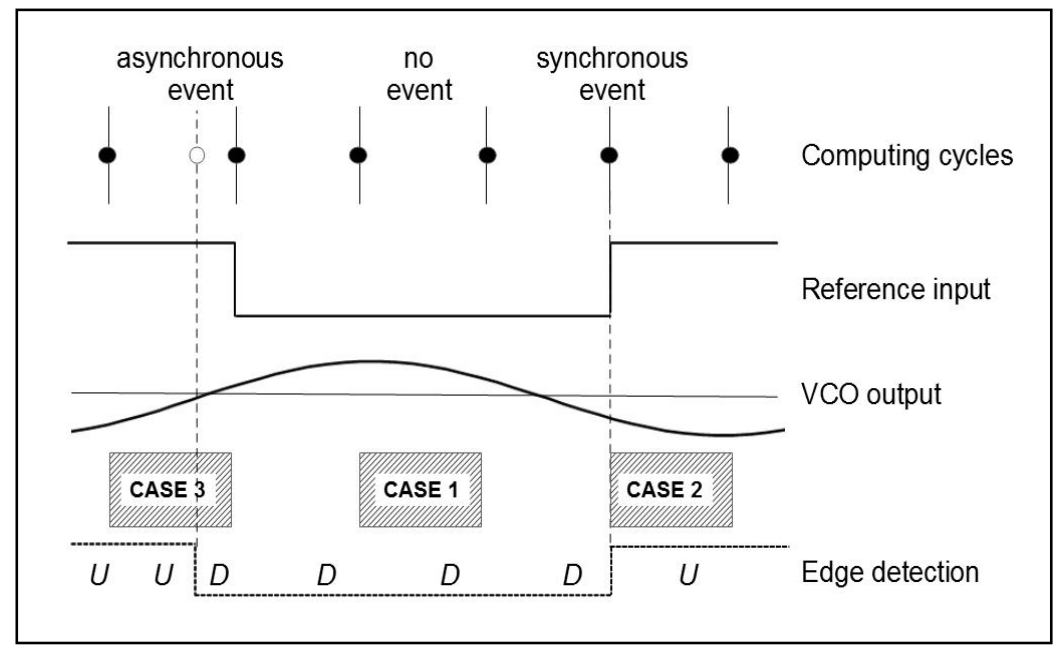

Figure 5 : Evènement asynchrone généré par le VCO

La fonction qui décrit le comportement de la CP-PLL le long d'un cycle est conçue, dans un premier temps, en supposant qu'il n'y a pas d'évènement asynchrone au milieu du cycle, et qu'il mesure l'état du système à la fin de celui-ci (Figure 6). Cette supposition correspond aux cas 1 et 2 décrits précédemment. Nous pouvons constater à la fin du cycle mesuré, que la sortie de la référence ne génère pas de changement dans l'état du PFD, et en conséquence sur la sortie du $\mathrm{CP}$. Lorsqu'un changement est identifié (cas 3), nous relancerons la détection en divisant le cycle en deux parties afin de prendre en considération ce changement à l'entrée du filtre de boucle. Avec ce type de scénario, la sortie du modèle est synchrone avec le cycle de mesure et, surtout, est compatible avec une simulation à commande par cycle.

Ce sera l'utilisation d'un outil efficace de transformée inverse de Laplace, dans la simulation du filtre de boucle LF, ce qui nous permettra l'exploration élargie de différentes configurations de notre système de boucle à verrouillage de phase CP-PLL. La solution proposée ici, qui est intégrée au puissant et versatile simulateur à signaux mixtes NAPA, est bien plus générique que le simulateur spécifique pour CP-PLL que nous avons proposé dans la référence [2], en plus d'être mieux adaptée à nos besoins. 


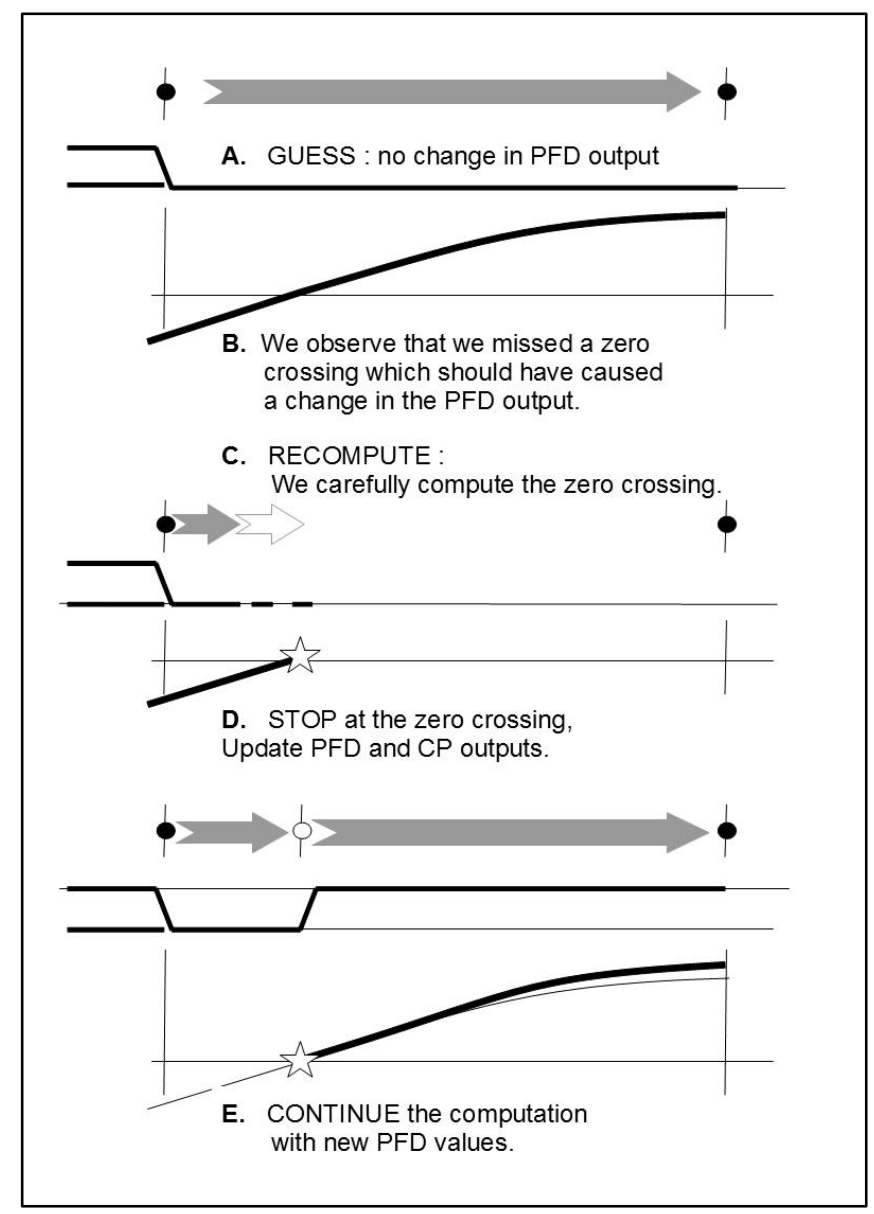

Figure 6 : Diagramme détaillé d'un évènement asynchrone (cas 3)

\section{Les travaux pratiques}

Nous avons conçu nos TPs comme des mini-projets où les étudiants doivent construire une CP-PLL à partir de composants de base. Dans un premier temps ils doivent acquérir une bonne compréhension du circuit et de ses caractéristiques comportementales. Les performances annoncées de ce type de circuit serviront d'indicateurs et définiront les objectifs de la CP-PLL qu'ils devront concevoir. Ils découvriront ensuite des techniques de montage sans soudure (câblage, débogage, découplage, ...) et devront développer quelques techniques de mesure (Figure 7).

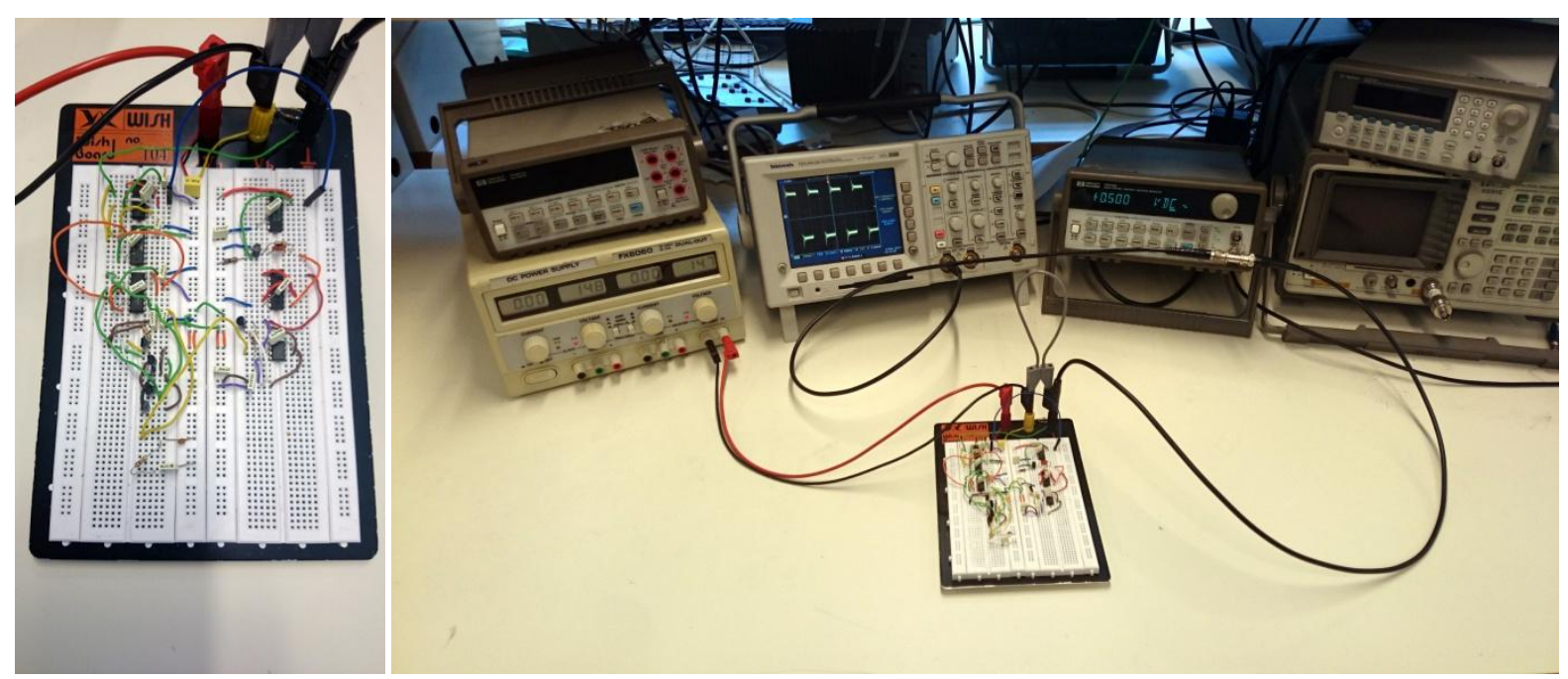

Figure 7: Banc de mesures pour le montage discret de la CP-PLL 
Ils commenceront alors leur design par un VCO constitué de composants discrets, ce qui va leur permettre de fixer la fenêtre de fréquences d'utilisation. Nous les guiderons en leur suggérant quelques schémas à considérer, comme l'exemple de la figure 8. Le VCO choisi est basé sur une bascule de Schmitt CMOS Hex CD40106B [8], qui dépend fortement de la tension d'alimentation, et d'un amplificateur opérationnel TL082. Le convertisseur de niveau est apparu comme difficile à dimensionner, ce qui nous a offert l'opportunité d'illustrer l'accumulation de charge sur un transistor bipolaire saturé, le ralentissement inhérent lorsqu'on l'éteint, et les techniques pour l'éviter.

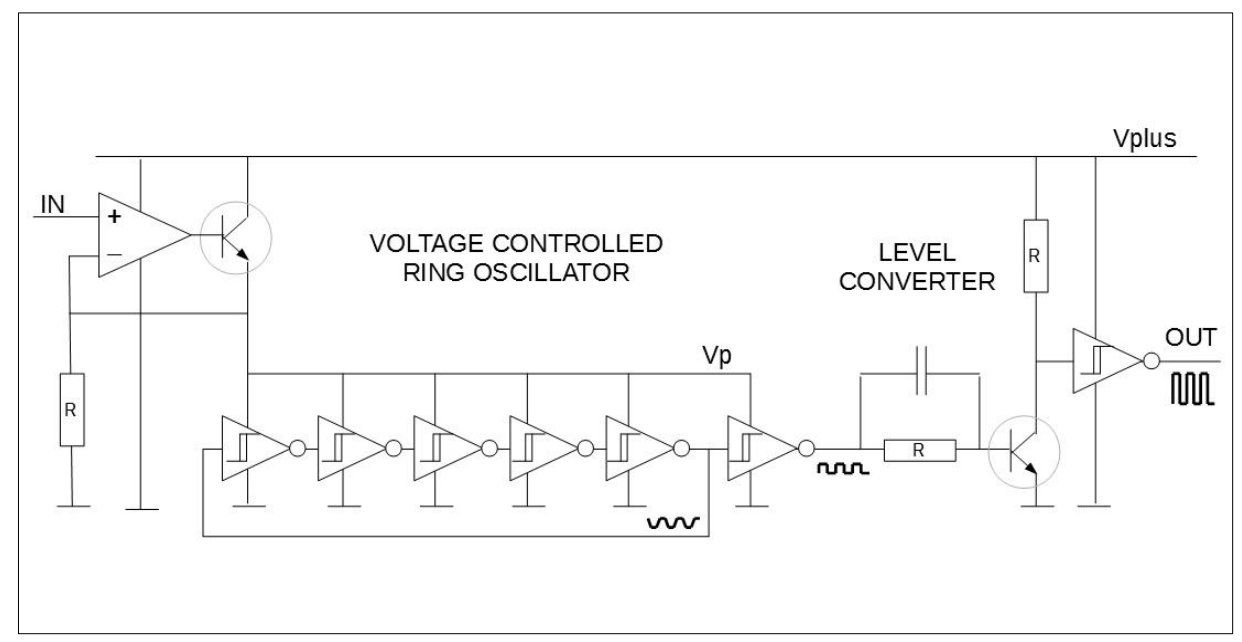

Figure 8: VCO et son convertisseur de niveau

La fonction de transfert Tension vers Fréquence $\mathrm{F}=\mathrm{f}(\mathrm{V})$ a été mesurée et enregistrée pour une utilisation ultérieure (Figure 9).

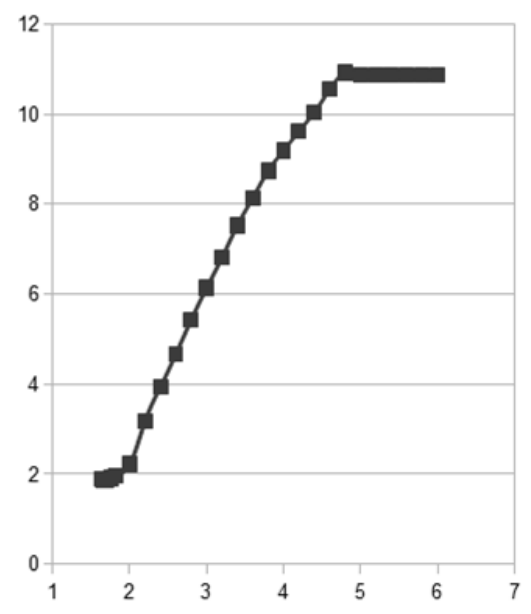

$$
\leftarrow \operatorname{Fout}(\mathrm{MHz}) \text { vs Vin(Volts) }
$$

Figure 9 : Mesures du montage VCO

Un exemple de pompe à charge alimentant un filtre de boucle est donné dans la figure 10. Les étudiants ont dû trouver des solutions compétitives. Les performances des sources de courant ont été simulées et comparées à celles du montage en laboratoire.

Le comparateur de phase PFD à trois états réalisé par les étudiants est montré sur la figure 11. Il utilise le circuit SN74HCT74N composé d'un double registre, où leur sorties sont rebouclées sur leur reset à travers une porte NAND. Ce système assure la génération d'un pulse (UP ou DOWN) qui démarre avec le premier signal (front montant) arrivant sur le PFD, et qui a une largeur égale au délai entre les deux signaux. 


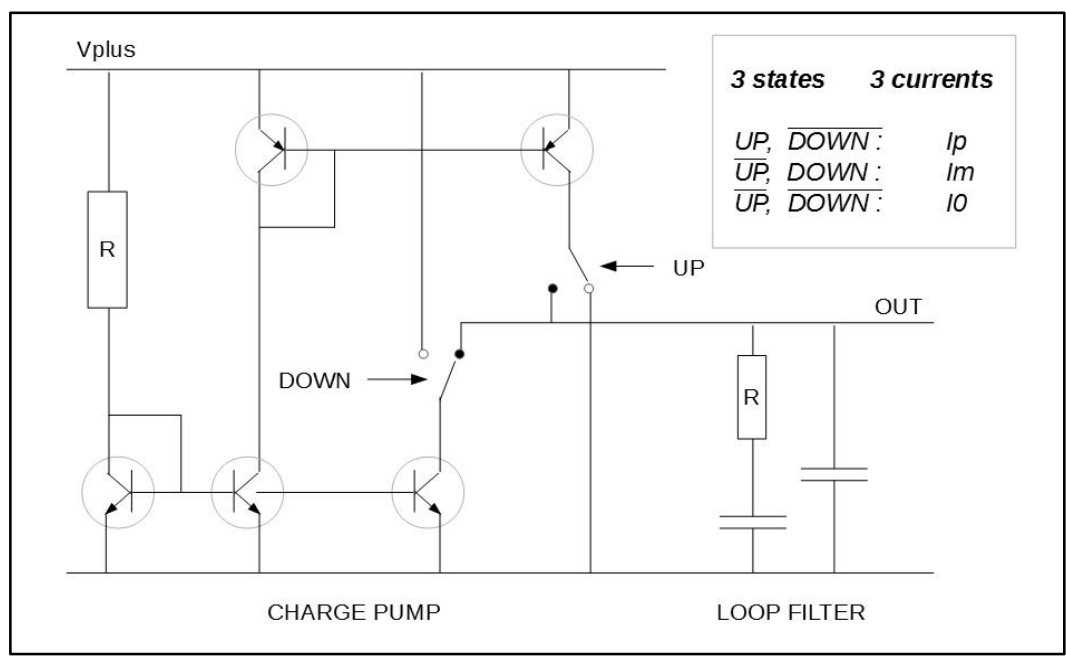

Figure 10 : Pompe à charge et Filtre à boucle

\section{Phase \& Frequency Detector}

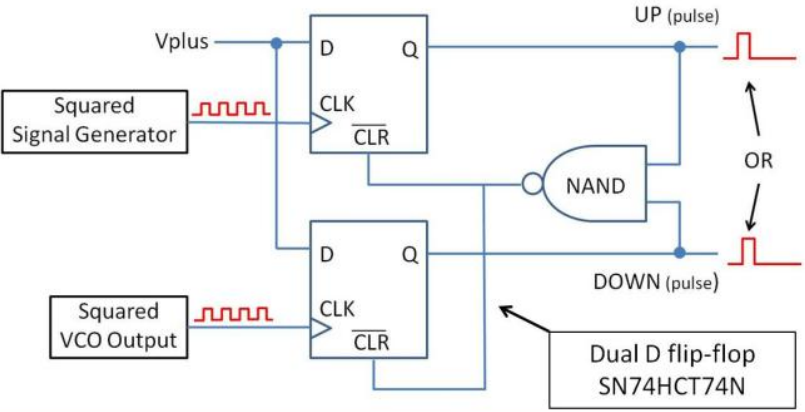

VCO slower than reference $\rightarrow$ UP pulse generated $\rightarrow$ Positive Current in CP $\rightarrow$ Charge of C into LP $\rightarrow$ Higher voltage supply on VCO $\rightarrow$ Speed Up of VCO

Figure 11 : Comparateur de phase PFD à 3 états

L'obtention de toutes les données importantes des sous-modules (Tableau 1) a permis la mise en place d'une simulation complète du système CP-PLL, puis l'optimisation du LF et la détermination de la meilleure architecture du PFD. Ci-dessous nous montrons un exemple de simulation pour 2 types de PFD (machine d'état à 3 ou 5 états), en utilisant les paramètres extraits de designs réalisés au niveau transistor en laboratoire :

\begin{tabular}{|c|c|c|}
\hline \multicolumn{2}{|c|}{$\begin{array}{l}\text { PFD } 3 \text { states: S1-, S0, S1+, } 5 \text { states: S2-, S1-, S0, S1+, S2+ } \\
\text { Delay Ref }=9.6 \mathrm{~ns} \\
\text { Delay Back }=10.6 \mathrm{~ns}\end{array}$} & $\begin{array}{l}\text { M, N divider ratios } \\
\begin{aligned} M & =1 \\
N & =1\end{aligned}\end{array}$ \\
\hline \multicolumn{3}{|l|}{ CP Currents, 3 levels: } \\
\hline$I-=-0.55 \mathrm{~mA}, \quad$ for PFD states $S 1-$ and S2- & P0 & $0 \mathrm{rad} / \mathrm{s}$ \\
\hline for PFD states SO & Pl & $=-45000 \mathrm{rad} / \mathrm{s}$ \\
\hline$I+=0.58 \mathrm{~mA}, \quad$ for PFD states $\mathrm{S} 1+$ and $\mathrm{S} 2+$ & Z0 & $=-5000 \mathrm{rad} / \mathrm{s}$ \\
\hline
\end{tabular}

\begin{tabular}{|l|} 
VCO Voltage to Frequency Piece Wise Linear Function: \\
$1.6 \mathrm{~V} \rightarrow 0.83 \mathrm{MHz}$ \\
$1.7 \mathrm{~V} \rightarrow 1.25 \mathrm{MHz}$ \\
$1.8 \mathrm{~V} \rightarrow 1.71 \mathrm{MHz}$ \\
$\ldots$ \\
$4.6 \mathrm{~V} \rightarrow 10.4 \mathrm{MHz}$ \\
$4.8 \mathrm{~V} \rightarrow 10.6 \mathrm{MHz}$ \\
$5.0 \mathrm{~V} \rightarrow 10.6 \mathrm{MHz}$
\end{tabular}

Tableau 1: Paramètres de simulation 
Les résultats de la simulation en sortie du VCO pour un signal de référence de type sinusoïdal à fréquence variable sont montrés sur la figure 12. Nous pouvons observer le temps de réponse non négligeable avant le verrouillage de phase, pour des changements brusques de la fréquence en entrée. On peut noter que cette simulation ne dure que $1,5 \mathrm{~s}$, et montre des résultats proches d'une simulation SPICE équivalente.

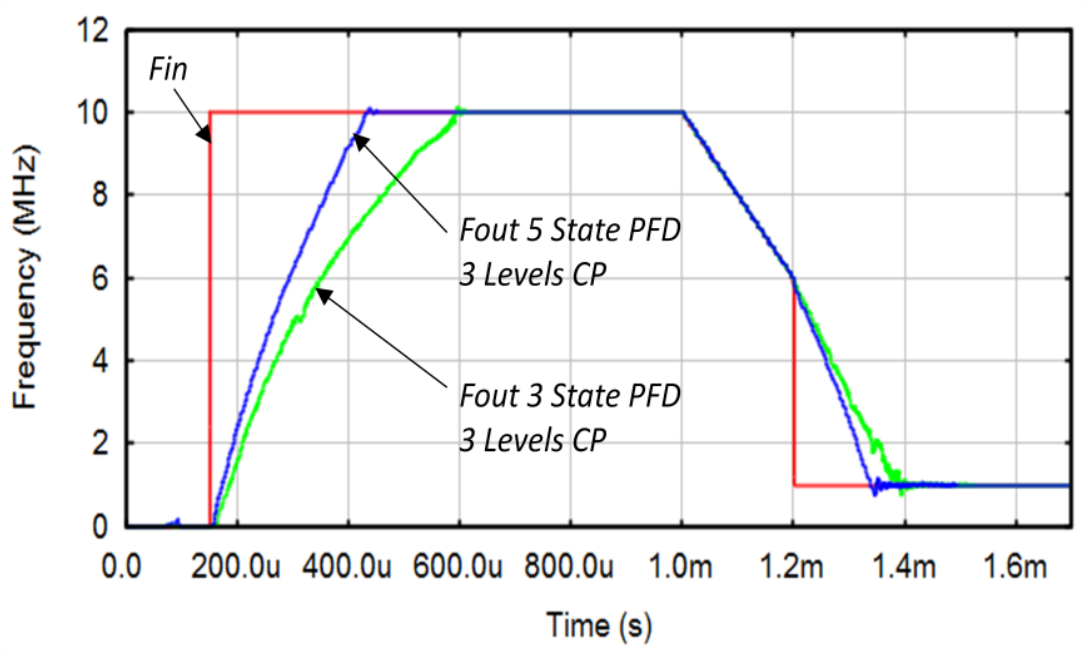

Figure 12 : Simulation réponse fréquentielle CP-PLL

Le challenge pour nos étudiants est de mesurer les performances de leur montage CP-PLL, ce qui n'est pas tache facile, et de les comparer avec celles obtenues par simulation, dans le but d'optimiser leur montage. Ils seront alors classés par la qualité et les performances de leur design.

L'étape finale consistera à se mesurer avec un circuit équivalent du marché, le «TI CMOS MicroPower PLL CD4046B » [9], à travers la caractérisation de celui-ci. Les étudiants doivent être capables maintenant d'identifier les points faibles de leur montage, ainsi que leurs atouts.

\section{Conclusion}

Cette nouvelle approche de travail offre à nos étudiants en micro-électronique l'opportunité de réaliser par eux-mêmes une CP-PLL à niveau transistor, tout en ayant acquis la compréhension de l'ensemble du système d'une façon progressive, par l'expérimentation et la modélisation/simulation. Ils sont censés obtenir les meilleures performances possibles de leur PLL et être capables d'intégrer leur modèle de simulation dans un système plus complexe, comme par exemple une chaîne de démodulation de signal radio FM. 


\section{Références}

[1] Miguel A. García Pérez, Yves Leduc and Fabien Ferrero, "Revamping a lab course for the education of students in electronic engineering", $6^{\text {th }}$ European Embedded Desing in Education and Research Conf., Sept 2014, Milan (ITALY), Proceedings of EDERC 2014 via IEEE Xplore \#9781-4799-6841-1, p. 162.

[2] F.M. Gardner, "Charge-Pump Phase-Lock Loops", IEEE Trans. Commun., Vol. COM-28, pp 1849-1858, Nov 1980.

[3] C.D. Hedayat, A. Hachem, Y. Leduc and G. Benbassat, "Modeling and Characterization of the 3rd Order Charge-Pump PLL : a fully event-driven approach", Analog Integrated Circuits and Signal Processing, Vol 19, Issue 1 pp 25-45, Apr. 1999.

[4] Wang, Ping-Ying, "Chaos In Phase Locked Loop", 2006 International Symposium on VLSI Design, Automation and Test, Hsinchu, Apr. 2006

[5] C.D. Hedayat, A. Hachem, Y. Leduc and G. Benbassat, "High-level modeling applied to the second order Charge-Pump PLL circuit", Texas Instruments Technical Journal, Vol 14, pp. 99-108, 1997.

[6] Yves Leduc, "NAPA, Outil de simulation comportementale de systèmes intégrés analogiques et mixtes", Mémoire en vue de l'obtention d'une HDR, Polytech'Nice-Sophia Antipolis, Jan. 2014.

[7] Napa is freely available for the education community (Contact the authors).

[8] CMOS Logic Gates, CD40106B, Texas Instruments

[9] CD4046B CMOS MicroPower Phase-Locked Loop, Texas Instruments.

\section{Biographies}

Miguel Angel García Pérez est né à Mollet del Vallès (España) en 1968. Maître es Sciences par l'Universitat de Barcelona. Thèse doctorale en Physique du Semi-conducteur par l'INSA de Lyon. Il a rejoint Texas Instruments France en 1997 où il a travaillé pendant 16 ans dans la conception de circuits intégrés. En 2013 il s'associe à Polytech'Nice-Sophia en tant que chargé d'enseignement vacataire, où il dispense des cours d'électronique. Il collabore avec l'unité de recherche EPOC dans le domaine de la détection et identification de signaux issues d'une boucle de Rogowski.

Yves Leduc, PhD, HDR, Chaire Texas Instruments à UNS. Après avoir obtenu un doctorat en sciences appliquees à l'UCL (Belgique) en 1979, Yves Leduc a rejoint Texas Instruments France en 1980. En 1993, il a formé le groupe de développement "Mixed Signal" et a été élu "TI Fellow" en 1998. Yves occupe la chaire industrielle TI à Polytech, Université de Nice Sophia-Antipolis. Son domaine d'activités principal est le design de circuits intégrés mixtes et la modélisation associée.

Fabien Ferrero est né à Nice (France) en 1980. Il a reçu son diplôme de thèse en 2007 à l'Université de Nice-Sophia Antipolis. Entre 2008 et 2009, il a travaillé pour la société IMRA Europe (centre de recherche d'Aisin Seiki) dans le domaine automobile. Après un Post-Doc au LEAT en 2009 sur le projet NAOMI, il est depuis 2010, Maître de Conférences à l'Ecole Polytech'Nice. Il effectue sa recherche au sein du LEAT. Ses études concernent la conception et la mesure d'antennes, de circuits déphaseurs et de systèmes reconfigurables. 